\title{
Plasmonic Lenses for Tunable Ultrafast Electron Emitters at the Nanoscale
}

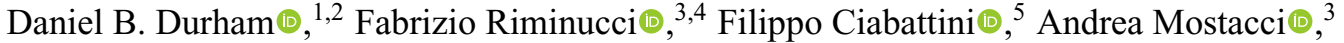 \\ Andrew M. Minor $\odot,{ }^{1,2}$ Stefano Cabrini, ${ }^{5}$ and Daniele Filippetto $\odot^{4, *}$ \\ ${ }^{1}$ Department of Materials Science and Engineering, University of California, Berkeley, Berkeley, \\ California 94720, USA \\ ${ }^{2}$ National Center for Electron Microscopy, Molecular Foundry, Lawrence Berkeley National Laboratory, \\ One Cyclotron Road, Berkeley, California 94720, USA \\ ${ }^{3}$ University of Rome "La Sapienza," Piazzale Aldo Moro 5, 00185 Rome, Italy \\ ${ }^{4}$ Accelerator Technology and Applied Physics Division, Lawrence Berkeley National Laboratory, One Cyclotron \\ Road, Berkeley, California 94720, USA \\ ${ }^{5}$ Molecular Foundry, Lawrence Berkeley National Laboratory, One Cyclotron Road, Berkeley, California 94720, \\ USA
}

(Received 8 July 2019; revised manuscript received 14 October 2019; published 25 November 2019)

\begin{abstract}
Simultaneous spatiotemporal confinement of energetic electron pulses to femtosecond and nanometer scales is a topic of great interest in the scientific community, given the potential impact of such developments across a wide spectrum of scientific and industrial applications. For example, in ultrafast electron scattering, nanoscale probes would enable accurate maps of structural dynamics in materials with nanoscale heterogeneity, thereby leading to an understanding of the role of boundaries and defects on macroscopic properties. On the other hand, advances in this field are mostly limited by the brightness and size of the electron source. We present the design, fabrication, and optical characterization of bullseye plasmonic lenses for next-generation ultrafast electron sources. Using electromagnetic simulations, we examine how the interplay between light-plasmon coupling, plasmon propagation, dispersion, and resonance governs the properties of the photoemitted electron pulse. We also illustrate how the pulse duration and strength can be tuned by geometric design and predict that sub-10-fs pulses with nanoscale diameter can be achieved. We then fabricate lenses in gold films and characterize their plasmonic properties using cathodoluminescence spectromicroscopy, demonstrating suitable plasmonic behavior for ultrafast nanoscale photoemission.
\end{abstract}

DOI: 10.1103/PhysRevApplied.12.054057

\section{INTRODUCTION}

Ultrafast electron sources have been extensively used as tools for scientific discovery over the past two decades. Such sources can now reliably produce electron pulses with femtosecond duration, which can be used as probes for dynamic microscopy and scattering measurements. Ultrafast electron-based measurements have provided insight into mechanisms of structural phase transitions in condensed matter [1-3] as well as chemical reactions and photochemistry in gases and molecular solids [4,5]. Even so, ultrafast electron experimentation is still limited by the source brightness, defined as the number density of electrons in transverse phase space [i.e., per unit solid angle and unit area, also called four-dimensional (4D) emittance] [6] and by setting a limit to beam relative coherence

\footnotetext{
*dfilippetto@lbl.gov
}

and focusability [7]. Spatiotemporal mapping with both nanoscale spatial and femtosecond temporal resolution is rarely utilized [8-10] and typically requires a combination of high-contrast signals, acquisition times of several hours, and limited sampling. Electron-based mapping of dynamics with increased throughput and detail may provide key insights into how microstructure and defects locally influence phase transformation, carrier generation and recombination, phonon and plasmon propagation, and much more.

In efforts to increase brightness, researchers have developed radio-frequency-based electron guns (rf guns) capable of sustaining large electric field amplitudes at extraction and during acceleration. By providing an orderof-magnitude-higher accelerating field $(100 \mathrm{MV} / \mathrm{m})$ than direct-current electron guns, the extracted peak current can be increased by orders of magnitude, from the microampere range typical of electron microscopes to several amps, while generating relativistic electron pulses with sub-10-fs 
duration [11,12]. Yet, tip emitters typically used in electron microscopes are not easily used in $\mathrm{rf}$ guns since, under such high fields, they have limited lifetimes and tend to emit dark electrons via field emission [13], which are not synchronous with the laser pulse and add background to the experiment. Instead, flat cathodes are often used, which have much larger emission areas in the range of tens of micrometers or larger, ultimately limited by the incident-laser spot size. Considering conservation of the beam emittance, a larger source size in turn produces a proportionally larger spot size for a given angular spread, thereby limiting the minimum useful spot size for applications.

Recently, nanostructured cathode surfaces have been demonstrated that couple the incident laser to resonant surface plasmon modes, which concentrate and enhance the optical-field intensity in localized areas of the surface. For example, nanogrooves designed to produce an in-groove surface plasmon resonance have been patterned into gold-coated cathodes and have been found to increase the photocurrent yield by 6 orders of magnitude over bare gold through multiphoton photoemission [14]. Nanohole arrays designed to have a grating resonance have also been demonstrated in copper cathodes, increasing the photocurrent yield by a factor of 120 over bare copper [15]. This approach to improving the emission characteristics is compatible with high accelerating fields. However, the photoemitted beams in these cases have still had limited quality [15]: the optical fields have been concentrated at edges, inducing emittance growth similar to the effect of surface roughness [16]. Also, their temporal response has not been studied in detail and may be limited by the cavityresonance damping time. In addition, the emission is still dispersed over areas of several microns in these initial demonstrations.

In this work, we propose and investigate the potential of bullseye plasmonic lenses for ultrafast nanoscale photoemitters. Such structures concentrate optical fields to a single central spot on a flat surface $[17,18]$, potentially providing aberration-free electron emission and enabling the use of nanoscale phototriggered emitters in high-field environments. We first show electromagnetic simulations to demonstrate how to control the spatiotemporal characteristics of the optical fields and the corresponding photoemission by geometric design. Then, we demonstrate the fabrication of actual bullseye lenses in gold films using two methods and characterize their plasmonic behavior using cathodoluminescence spectromicroscopy. We show that spatial and spectral plasmonic characteristics are as predicted in simulation, supporting the notion that ultrafast nanoemission can be achieved. Altogether, the results support the potential for bullseye lenses as highbrightness electron sources and establish a research direction in the field of plasmon-enhanced ultrafast electron nanoemission.

\section{PLASMONIC-LENS DESIGN AND ELECTROMAGNETIC SIMULATIONS}

\section{A. Lens principle and parameters}

The system under study is a nanopatterned photocathode excited by ultrafast laser pulses with an 800 -nm center wavelength. The pattern consists of equally spaced concentric annular grooves, forming a bullseye plasmonic lens. The geometry is defined by the five parameters illustrated in Fig. 1(a): the number of rings $N$, the grating period $p$, the groove width $w$, the groove depth $d$, and the center-plateau radius $r_{i}$. Such gratings couple the component of the incident light with the electric field perpendicular to the grooves into surface plasmon polaritons

(a)

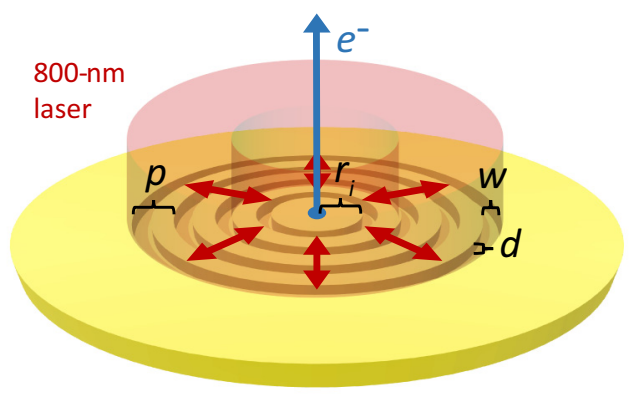

(b)

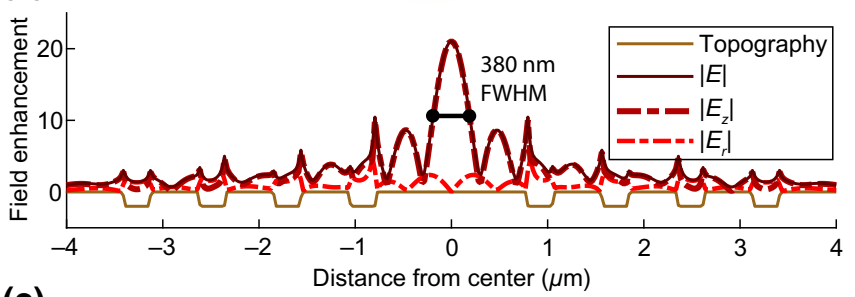

(c)

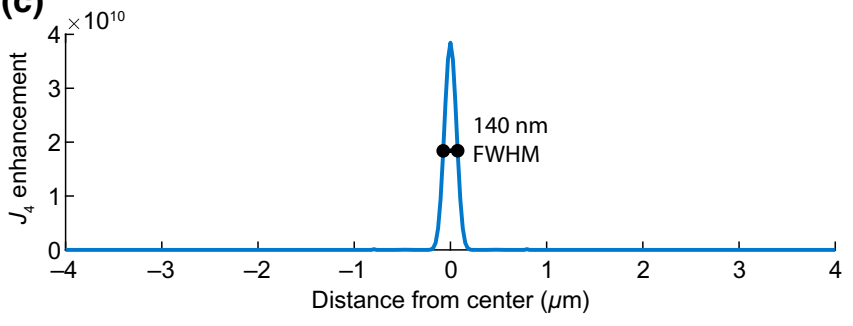

FIG. 1. The simulated electric field and photoemission profiles during excitation of a Au plasmonic bullseye lens with a radially polarized 800-nm continuous-wave (cw) laser. (a) A schematic of the bullseye geometry. The parameters shown include the grating period $p$, the groove width $w$, the groove depth $d$, and the center-plateau radius $r_{i}$. For the simulation results in the following subpanels, $p=783 \mathrm{~nm}, d=90 \mathrm{~nm}, w=270 \mathrm{~nm}$, and $r_{i}=783 \mathrm{~nm}$. The number of rings $N=4$. (b) The total $(|E|)$ electric field enhancement, shown with its normal $\left(\left|E_{z}\right|\right)$ and tangential $\left(\left|E_{r}\right|\right)$ components at the bullseye surface. The field enhancement is defined relative to the peak field in the incident beam. The bullseye topography is superimposed on the plot for reference: the grooves are $90 \mathrm{~nm}$ deep. (c) The calculated four-photon photocurrent-density enhancement $\left(J_{4}\right)$ profile at the bullseye surface, using the generalized Fowler-Dubridge equation [19]. FWHM, full width at half maximum. 
(SPPs). A radially polarized laser at normal incidence is used so that the electric field direction is always perpendicular to the grooves and the launched SPPs are in phase. These SPPs then propagate and interfere to give maximum field enhancement at the structure center.

We use gold as the plasmonic material, enabling fourphoton photoemission at $800-\mathrm{nm}$ wavelength. Gold is oxidation resistant and provides effective photoemission surfaces; in fact, four-photon photoemission from gold cathodes patterned with linear gratings has been demonstrated [14]. Gold also provides long SPP propagation lengths in the red and near-infrared range: $10-15 \mu \mathrm{m}$ in as-deposited polycrystalline films [20,21] and greater than $60 \mu \mathrm{m}$ in template-stripped and single-crystal gold films [21-23].

\section{B. Nanoscale field enhancement and photoemission}

We first simulate the electromagnetic fields for a lens under $\mathrm{cw}$ illumination to study their spatial distribution and the expected emission spot size. An 800-nm-wavelength laser is focused at the surface using a numerical aperture (NA) of 0.07 , giving a donut-shaped in-plane intensity with a peak-to-peak diameter of $4.5 \mu \mathrm{m}$. The lens has four rings with period $p$ of $783 \mathrm{~nm}$, which is the corresponding SPP wavelength in gold. This aims to satisfy momentum conservation between the normal-incidence photons and the SPPs as given by the grating equation [24]:

$$
\mathbf{k}_{\mathrm{spp}}=\mathbf{k}_{\mathrm{photon}, x y} \pm n \mathbf{g} .
$$

Here, $\mathbf{k}_{\text {spp }}$ and $\mathbf{k}_{\text {photon }}$ are the in-plane wave vectors of the SPP and incident photon, respectively, while $\mathbf{g}$ is the grating vector and $n$ is an integer. We set $r_{i}$ to be $783 \mathrm{~nm}$ to coincide with antinodes of the two-dimensional (2D) standing wave formed by the interfering SPPs. This causes reflections from the edge to resonate, further increasing the field enhancement. With these parameters fixed, we then perform a series of finite-difference time-domain (FDTD) simulations in LUMERICAL [25] to optimize the depth and width of the rings for maximum field enhancement at the center. The optimum is found for $d=90 \mathrm{~nm}$ and $w=$ $270 \mathrm{~nm}$.

The electric field magnitude profiles for the optimized structure are shown in Fig. 1(b). The field enhancement relative to the peak field of the incident laser is maximized at the center (21.0). Also, the lateral electric field $E_{r}$ is zero at the center and remains small relative to the normal field $E_{z}$ within the four-photon photoemission peak shown in Fig. 1(c).

The anticipated four-photon photocurrent density $J_{4} \propto$ $|\mathbf{E}|^{8}$ [19]; the photocurrent density enhancement is shown in Fig. 1(b). Remarkably, the $J_{4}$ enhancement at the center is $3.8 \times 10^{10}$. Also, the use of multiphoton photoemission practically eliminates contributions from the side lobes and edges of the structure, creating a single tightly focused emission spot with a full width at half maximum (FWHM) of $140 \mathrm{~nm}$ in the flat center plateau.

\section{Ultrafast temporal response}

To study the emitter's temporal response and how it depends on the lens geometry, we perform impulseresponse FDTD simulations. Using incident laser pulses with a 3-fs FWHM duration, we simulate the time-resolved electric field at the structure center, $E_{z, \text { center }}(t)$. The spectral response in the linear intensity regime is described by the complex transfer function $T(\omega)$, obtained from the Fourier transforms of the time-dependent incident and enhanced fields [26]:

$$
E_{z, \text { center }}(\omega)=T(\omega) E_{x, \text { inc }}(\omega) .
$$

The frequency-dependent field enhancement is then given by $|T(\omega)|$. We also use Eq. (2) to compute the temporal response for Gaussian incident pulses of varying duration.

We first study the four-ring bullseye lens optimized above (see Fig. 1). The simulated incident and enhanced electric fields over time are shown in Fig. 2(a). Notably, the plasmonic field is stronger than the incident laser field but lasts more than 10 fs longer. In the next section, we show that some of this broadening is due to the delay in arrival of plasmons generated from outer rings relative to inner rings. This leads to a spectral response with a finite bandwidth, shown in Fig. 2(b) as a function of the incident-photon wavelength. There is a strong peak at 800 $\mathrm{nm}$, which we attribute to a surface plasmon resonance in the center plateau. The grooves in this structure are deep enough to disturb SPP propagation and modify their dispersion, leading to additional peaks and valleys in the response (see Fig. 4). Additional peaks have been observed experimentally in transmission spectra of plasmonic lenses with a central aperture [27] and computational work finds significant dispersion modification in deep gratings, such as the opening of plasmonic band gaps [28].

Effects on the response shape for varying incidentpulse length $\left(I_{\text {inc }}\right.$ FWHM) are shown in Fig. 2(c). Longer pulses generate a Gaussian response with a peak field enhancement identical to that for $\mathrm{cw}$ illumination. On the other hand, for pulse lengths of tens of femtoseconds, there is substantial temporal broadening and the plasmonic field amplitude is reduced. These effects on the response strength and duration are quantified in Figs. 2(d) and 2(e). We note that the FWHM durations in Fig. 2(e) are set by the main peak in the temporal envelope, while the tails are suppressed due to the scaling of four-photon photoemission with the intensity $\left[J_{4}(t) \propto I^{4}(t)\right][19]$. As a result, the response duration is mostly linear with the input-pulse duration and always about 3.5 fs longer due to the propagation delay between rings. For few-femtosecond pulse lengths, there are deviations from linearity that may 
(a)

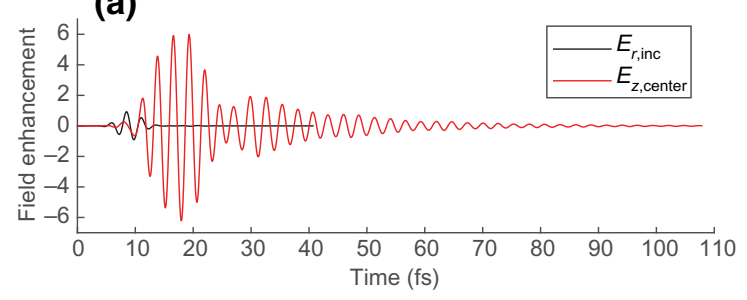

(c)

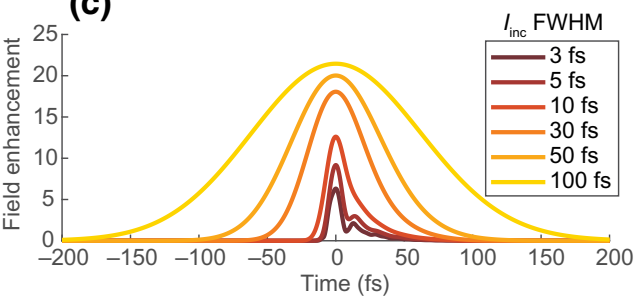

(d)

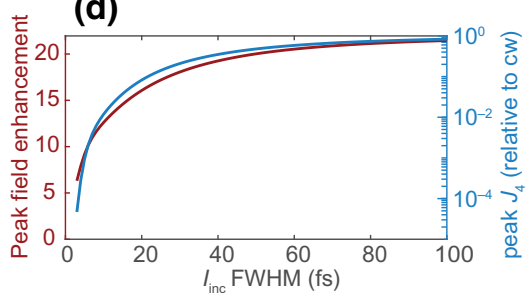

(b)

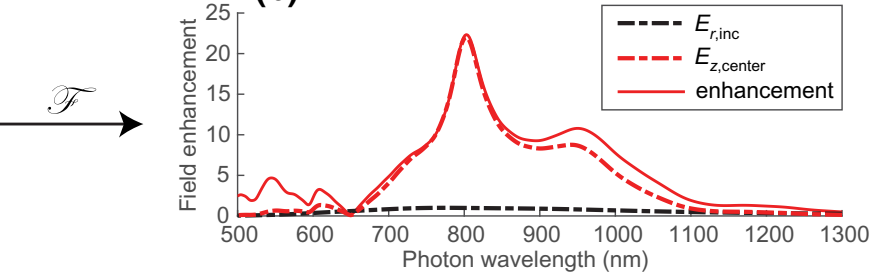

(e)

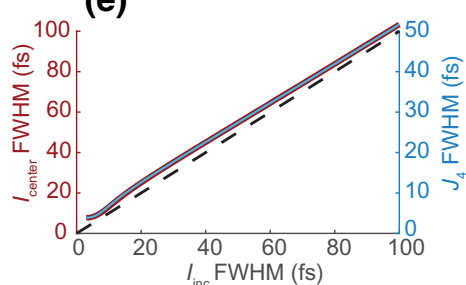

FIG. 2. The simulated ultrafast temporal response of the Au plasmonic bullseye lens studied in Fig. 1 . The number of rings $N=4$, the grating period $p=783 \mathrm{~nm}$, the groove width $w=270 \mathrm{~nm}$, the groove depth $d=90 \mathrm{~nm}$, and the center-plateau radius $r_{i}=783 \mathrm{~nm}$. (a) The impulse response computed using an incident radially polarized laser pulse with a temporal FWHM of 3 fs. $E_{r \text {,inc }}$ is the maximum lateral electric field of the incident pulse and $E_{z \text {,center }}$ is the normal electric field at the bullseye center. The electric field is normalized to the maximum $E_{r \text {,inc }}$. (b) The transfer function obtained by Fourier transformation of the impulse response. The enhancement, i.e., the magnitude of the transfer function, is shown along with the magnitudes of the Fourier transforms of $E_{r \text {,inc }}$

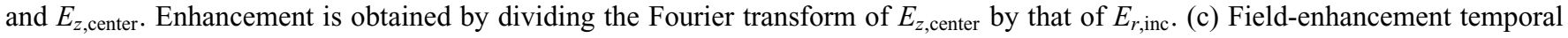
envelopes for varying incident-pulse durations. $I_{\text {inc }}$ FWHM is the temporal FWHM of the incident-pulse intensity. (d) The peak field enhancement and photocurrent density $\left(J_{4}\right)$ at the bullseye center as a function of the incident-pulse duration. The peak $J_{4}$ is plotted relative to the value of $J_{4}$ under $\mathrm{cw}$ illumination. The tops of both $y$ axes correspond to the value under cw illumination. (e) The temporal FWHM of the field intensity at the bullseye center $\left(I_{\text {center }}\right)$ and $J_{4}$ as function of the incident-pulse duration.

be related to beating and envelope asymmetry. Still, this lens is predicted to be capable of producing sub-10-fs photoelectron pulses.

\section{Tuning the response by geometric design}

We now present a series of impulse-response simulations while varying geometric parameters to clarify design rules for ultrafast photoemission applications. We first vary the number of rings, $N$, obtaining the simulated spectral response shown in Fig. 3(a). For $N=1$, the bandwidth extends over the entire wavelength range studied and there is a plasmonic-resonance peak at $800 \mathrm{~nm}$. Adding more rings increases the field enhancement by coupling more light but it shrinks the bandwidth by increasing the SPP propagation distance, and hence the delay time, between the inner and outer rings. This generally increases the peak field enhancement for the varying incident-pulse length shown in Fig. 3(b) while increasing the response duration, as shown in Fig. 3(c). For few-femtosecond pulses, however, there is a limit to the number of added rings that increase field enhancement, beyond which the delay time between the inner and outer plasmons is too long for them to overlap. In this limit, adding additional rings only increases the pulse duration without increasing the field enhancement, as shown in Figs. 3(b) and 3(c) at the shortest pulse durations.
We then vary the depth of the grooves, $d$, obtaining the spectral response shown in Fig. 4(a). As $d$ increases, the field enhancement increases, saturates, and eventually decreases. While deeper grooves couple more incident photons to SPPs, they also inhibit SPP propagation to the center, leading to an optimal depth that maximizes field enhancement. The resonance peak at $800 \mathrm{~nm}$ emerges and grows with increasing $d$ as reflectivity of plasmons from the grooves increases. Other peaks and valleys also emerge, suggesting that the deeper grooves are disturbing plasmon propagation and modifying their dispersion and interference. The peak field enhancement for varying incident-pulse length scales similarly with $d$, as shown in Fig. 4(b). Again, for few-femtosecond pulses, plasmons from the outer rings cannot reach those from the inner rings and so the field enhancement is reduced. There is also a noticeable effect of $d$ on the temporal-response duration as shown in Fig. 4(c). For longer pulses, the duration seems to scale with the strength of the resonance contribution, which would extend the plasmonic field duration. Overall, $d$ has less of an effect on the pulse duration than $N$.

Next, we vary the ring width, $w$, computing the transfer functions shown in Fig. 5(a). Maximal field enhancement is achieved for $w=360 \mathrm{~nm}$, close to half the period. Adjusting the width also shifts the resonance peak. Wider groove obstacles may more strongly obstruct plasmon propagation, affecting the plasmon dispersion 
(a)

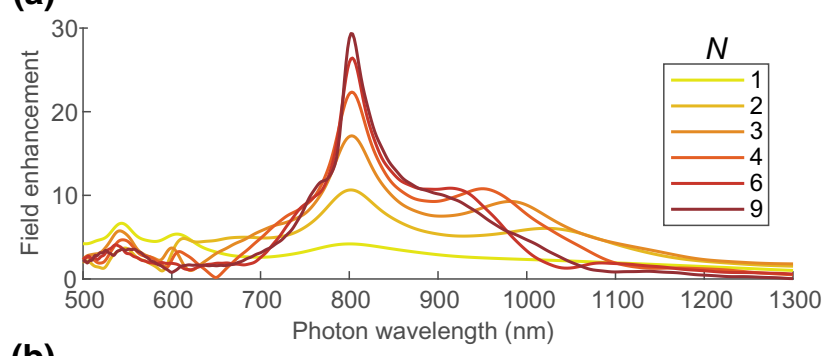

(b)

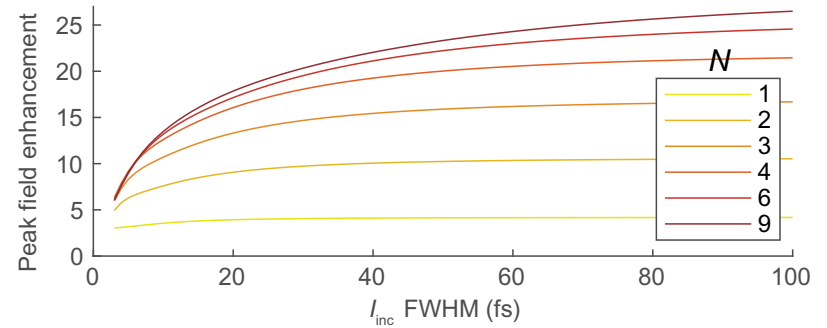

(c)

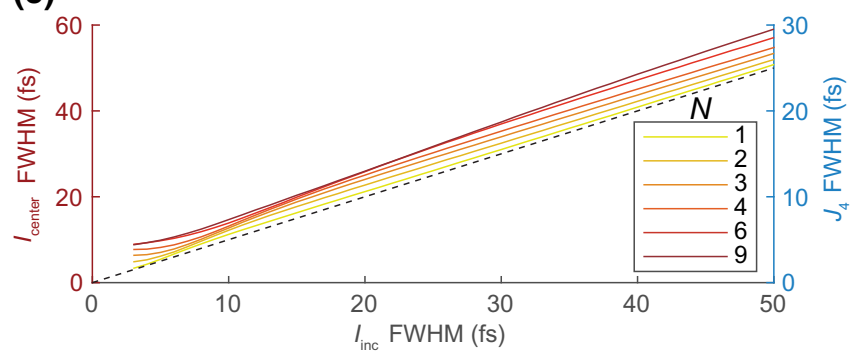

FIG. 3. The simulated ultrafast temporal response of bullseye lenses with varying numbers of rings, $N$. The grating period $p=783 \mathrm{~nm}$, the groove width $w=270 \mathrm{~nm}$, the groove depth $d=90 \mathrm{~nm}$, and the center-plateau radius $r_{i}=783 \mathrm{~nm}$. (a) The transfer function computed for lenses with $N$ ranging from 1 to 9 . (b) The maximum field enhancement at the lens center for incident pulses with a varying temporal FWHM. (c) The temporal FWHM of the electric field intensity at the lens center $\left(I_{\text {center }}\right)$ for incident pulses with a varying temporal FWHM. The temporal FWHM of the four-photon photocurrent density $J_{4}$ is half of the $I_{\text {center }}$ FWHM.

relation. This shift in resonance peak can be compensated for by adjusting the bullseye grating period, $p$. The transfer function and temporal response of a four-ring lens optimized by allowing variable $p$ is shown with that obtained by fixing $p=783 \mathrm{~nm}$ in Fig. 5(b). The peak field enhancement and the symmetry of the transfer function are improved by using $w=360 \mathrm{~nm}$ and compensating for the resonance peak shift by setting $p=760 \mathrm{~nm}$. This provides a factor-of-4 increase in the estimated four-photon photoemission yield. This comes without cost in the response duration, as shown in Fig. 5(c).

These results lead to a few design rules for ultrafast applications. The depth and width of the grooves should usually be optimized for maximum field enhancement. The period can then be adjusted to center the resonance peak at the desired wavelength. Finally, the structure should (a)

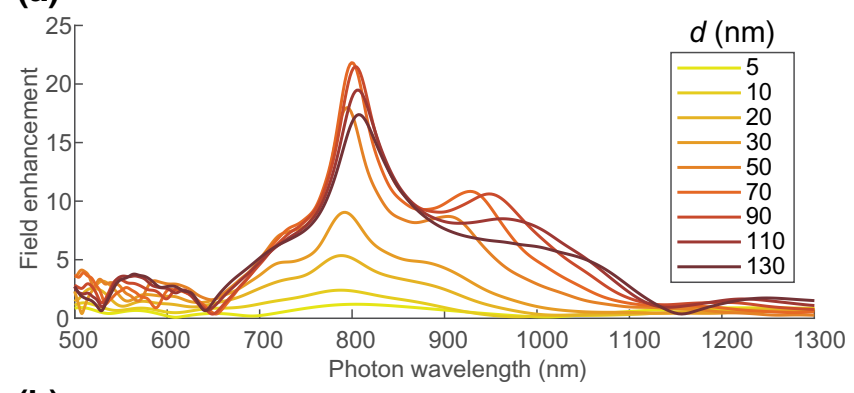

(b)
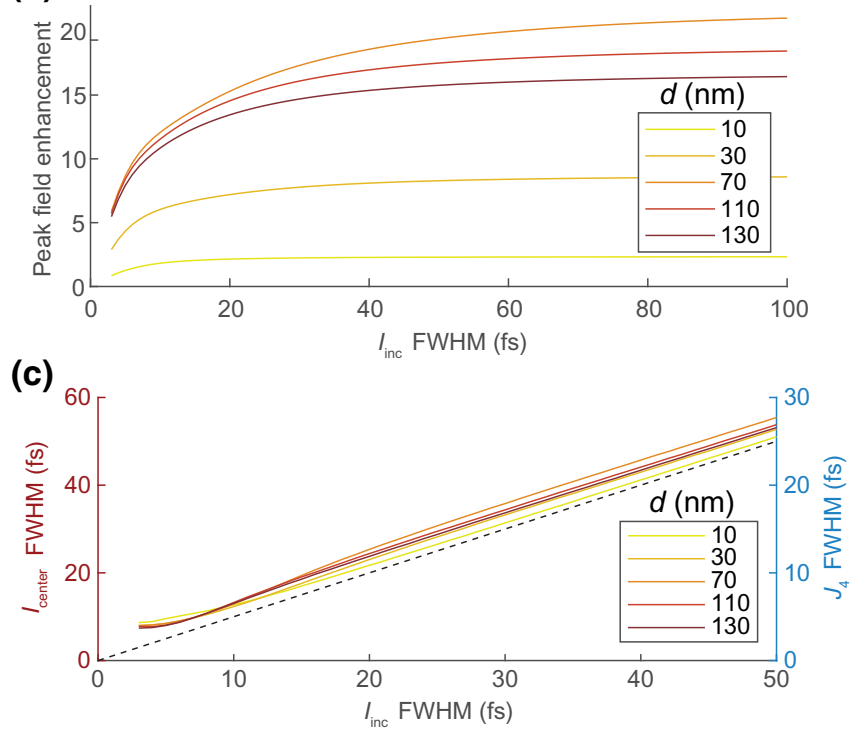

FIG. 4. The simulated ultrafast temporal response of bullseye lenses with varying groove depth, $d$. The number of rings $N=4$, the grating period $p=783 \mathrm{~nm}$, the groove width $w=270 \mathrm{~nm}$, and the center-plateau radius $r_{i}=783 \mathrm{~nm}$. (a) The transfer function computed for lenses with varying $d$. (b) The maximum field enhancement at the lens center for incident pulses with a varying temporal FWHM. (c) The temporal FWHM of the electric field intensity at the lens center $\left(I_{\text {center }}\right)$ for incident pulses with a varying temporal FWHM. The temporal FWHM of the four-photon photocurrent density $J_{4}$ is half of the $I_{\text {center }}$ FWHM.

have as many rings as possible to maximize the photocurrent while maintaining enough bandwidth to achieve the required pulse duration. The optimal geometry ultimately depends on the photocurrent and pulse duration required.

\section{FABRICATION}

We fabricate bullseye lenses using two processes illustrated in Fig. 6. One process involves thermally evaporating $5 \mathrm{~nm}$ of titanium as an adhesion layer and 150 $\mathrm{nm}$ of gold onto a Si wafer, then carving out the rings using focused-ion-beam (FIB) milling. A Zeiss Crossbeam $1540 \mathrm{EsB}$ is used for the FIB. The other process uses electron-beam lithography followed by template stripping to produce high-precision smooth cathode surfaces [29]. 


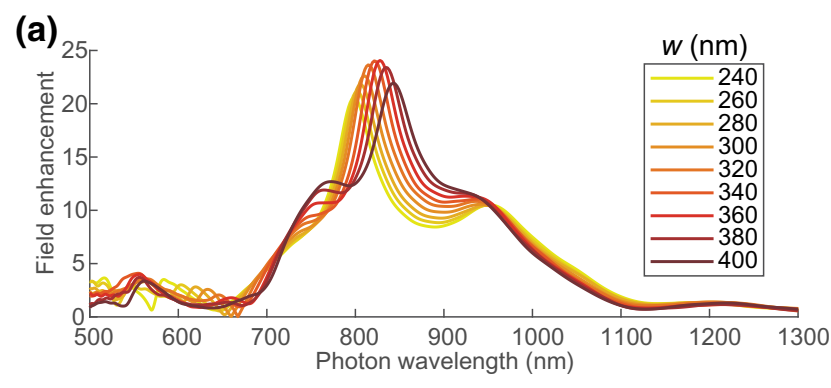

(b)

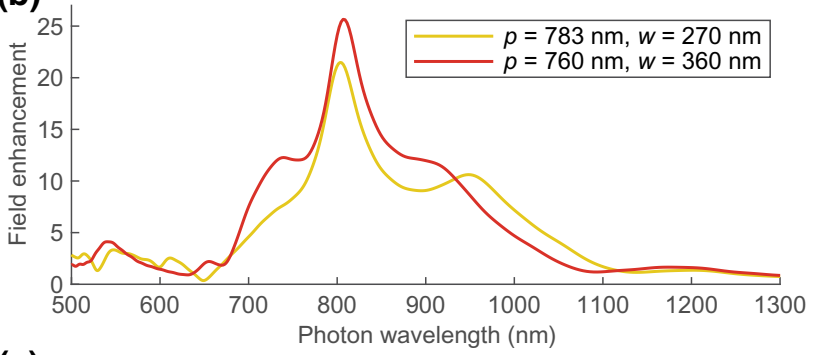

(c)

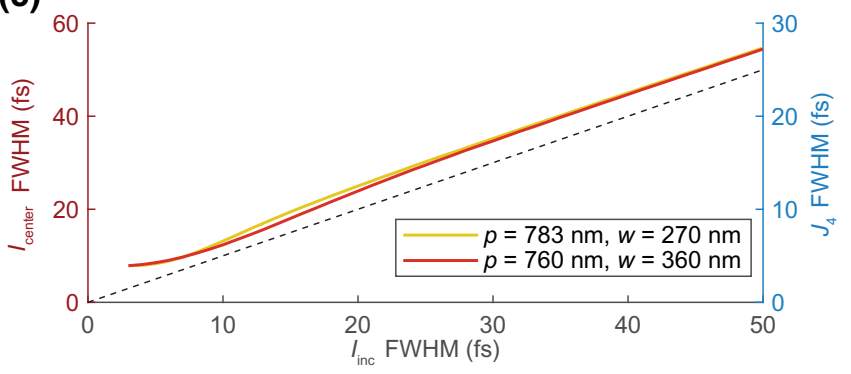

FIG. 5. The simulated ultrafast temporal response of bullseye lenses with varying groove width, $w$. The number of rings $N=4$, the groove depth $d=90 \mathrm{~nm}$, and the center-plateau radius $r_{i}=783 \mathrm{~nm}$. (a) The transfer function computed for lenses with varying $w$ and grating period $p=783 \mathrm{~nm}$. (b) The transfer function computed for the lens studied in Fig. 1 ( $p=783 \mathrm{~nm}$, $w=270 \mathrm{~nm}$ ) and for a lens with $w$ chosen to maximize field enhancement and $p$ adjusted to center the peak wavelength near $800 \mathrm{~nm}(p=760 \mathrm{~nm}, w=360 \mathrm{~nm})$. (c) The response duration for the two lenses in (b). This is plotted as temporal FWHM of the electric field intensity at the lens center $\left(I_{\text {center }}\right)$ for incident pulses with a varying temporal FWHM. The temporal FWHM of the four-photon photocurrent density $J_{4}$ is half of the $I_{\text {center }}$ FWHM.

A negative e-beam resist hydrogen silsesquioxane (HSQ) $2 \%$ is spun at $1000 \mathrm{rpm}$ onto a $\mathrm{Si}$ wafer. The resist is then exposed using a Vistec VB300 electron-beam lithography system and developed, leaving the designed pattern in the form of amorphous silica on the wafer. Then, 150 $\mathrm{nm}$ of gold is deposited onto the template, entirely covering it. Finally, the patterned gold is peeled off using an electrically and thermally conductive ultra-high-vacuumcompatible epoxy resin. atomic force microscopy (AFM) measurements confirm that similar groove depths can be made in both structures, but the e-beam lithography (EBL) and template stripping process yields smoother surfaces

\section{(a)}

Focused ion beam

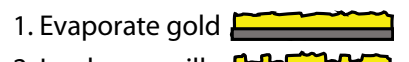

2. lon-beam mill

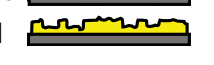

(b)

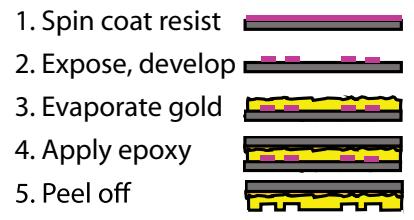

(c)

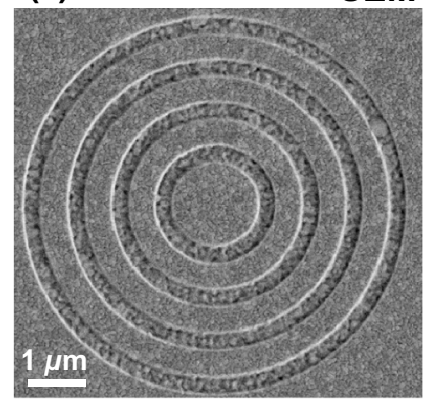

(d)

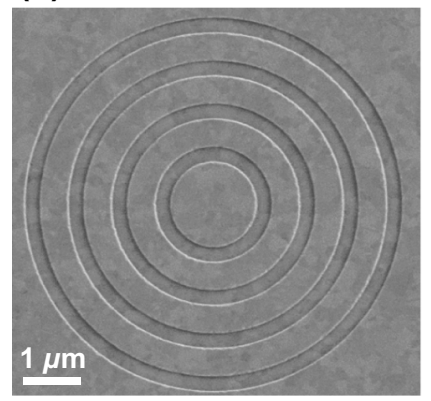

(e)

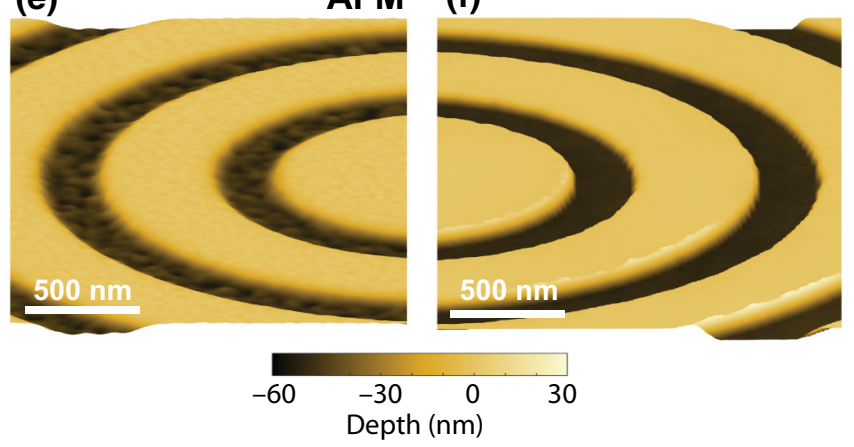

FIG. 6. Bullseye lenses fabricated using two methods. Process steps shown for (a) FIB milling and (b) EBL with template stripping. In-lens scanning-electron-microscopy (SEM) images of the bullseye lenses, made using (c) FIB and (d) EBL. AFM surfacetopography maps of lenses, made using (e) FIB and (f) EBL. The maps are displayed to scale in three-dimensional (3D) at a $40^{\circ}$ tilt.

and grooves. In fact, structures made by EBL have nearly atomically flat central areas, which minimizes degradation of the emitted electron beam from surface roughness and imperfections [16] (see Table I). In these lenses, the gratings are $50 \mathrm{~nm}$ deep, with parameters otherwise matching the first case (783-nm period, 270-nm width, four rings): its simulated spectral response is shown in Fig. 4.

TABLE I. A topographic comparison between EBL and FIB bullseye lenses via AFM.

\begin{tabular}{lcc}
\hline \hline & FIB & EBL \\
\hline Groove depth (nm) & 51 & 54 \\
Center rms roughness (nm) & 1.4 & 0.4 \\
Groove rms roughness (nm) & 3.2 & 0.6 \\
\hline \hline
\end{tabular}




\section{CATHODOLUMINESCENCE SPECTROMICROSCOPY}

\section{A. Examining plasmonic properties}

Here, we present cathodoluminescence- (CL) spectromicroscopy measurements of the plasmonic characteristics of the fabricated lenses. In CL, an electron beam is focused on the sample and, by one or more mechanisms, light is emitted from the material [30]. For plasmonic structures, the relevant mechanism is the broadband generation of SPPs by the fast-moving electrons as they strike the material surface. The SPPs propagate radially outward from a nanometric spot, which can then couple out to light through the bullseye grating [31,32]. When the electron beam is at the bullseye center, circularly symmetric SPPs are excited like those that would be generated by a radially polarized laser.

We use CL to measure a few key plasmonic properties of these structures. For one, the plasmonic resonance in the central region can be mapped using CL spectromicroscopy. CL intensity has been linked to the radiative local density of optical states (LDOS) at the electron-beam position, i.e., the number of optical modes available at the excitation position that produce light $[31,33]$. The LDOS is enhanced by plasmonic resonance, so CL can be used to spatially and spectrally resolve resonance modes [34,35]. Spatial homogeneity, circular symmetry, and a strong, sharp central peak are desirable for photoemission applications. The center wavelength and bandwidth of this resonance can be extracted from the CL spectrum obtained at the structure center. Also, the radial extent and circular symmetry of plasmon propagation and grating coupling can be inferred by angle-resolved imaging of the CL far field, which is essentially a Fourier transform of the real-space emission profile of the structure. These are important to characterize since significant propagation losses or asymmetries in coupling would reduce the field enhancement under laser illumination.

We use a modified Zeiss Gemini SUPRA 55 SEM for our CL measurements. The sample is positioned at the focal point of a horizontal $\mathrm{Al}$ parabolic mirror with 1-mm focal length. A $10-\mathrm{keV}$ electron beam is focused onto the sample and the emitted light is collected by the parabolic mirror over a wide angle range $\left(0^{\circ}-80^{\circ}\right.$ from normal $)$ and over the entire visible spectrum and beyond. The sample is tilted by about $25^{\circ}$ so that the highly directed normal emission will not escape through the entry hole in the mirror for the electron beam. We do not expect this tilt to change the plasmonic response; there will not be any significant arrival-time delay across the electron beam since it is much smaller than the plasmon wavelength. Also, the electron beam only generates SPPs at the surface, so the source size is only made about $10 \%$ larger along the tilt direction.

\section{B. CL spectromicroscopy of plasmonic resonance}

We use scanning CL spectromicroscopy to probe the spectral and spatial characteristics of the surface-plasmon resonance in our fabricated lenses. The electron beam is rastered step by step over the central plateau of the structure and a full CL-emission spectrum is collected at each beam position. Spectra are obtained by focusing the reflected light from the mirror onto a multimode optical fiber with a $200 \mu \mathrm{m}$ diameter and then dispersing it using a spectrometer consisting of an Acton 2300i monochromator (150 lines/mm, $500 \mathrm{~nm}$ blazed grating) and an Andor Newton electron-multiplied charge-coupled device (CCD). The dark current background is subtracted and spectra are normalized by the instrument response over the measured wavelength range. The open-source PYTHON-based SCOPEFOUNDRY software developed to control this experiment is available online for further reference [36,37].

From this 3D data set, we can extract average emission spectra from regions of interest. Average spectra over the region within $75 \mathrm{~nm}$ of the bullseye center are shown in Fig. 7(a). The spectra shown here are smoothed using a second-order Savitsky-Golay filter with a 15-nm window. The CL emission spans the entire detection spectral range. This is because a SPP of any wavelength from the broadband range generated by the electron beam can satisfy the grating equation and couple to light at a wavelengthdependent emission angle, and nearly all emission angles are collected by the parabolic mirror. However, there is a notable emission peak near $800 \mathrm{~nm}$, where a surface plasmon resonance is expected.

We can then examine the spatial profile of the plasmonic resonance. We apply virtual Gaussian band-pass filters with $2 \sigma=10 \mathrm{~nm}$ to the entire data set, yielding maps of CL emission over narrow spectral bands as a function of the beam position [see Figs. 7(c)-7(j)]. For maps of emission near the resonance wavelength, a zero-order Bessel-function spatial profile is observed corresponding to a cylindrical plasmonic resonance of the central plateau. For the maps at wavelengths just outside of the resonance peak, such a spatial profile is not observed, confirming that it is a resonance effect. For the structure made by EBL, the $\mathrm{CL}$ intensity is higher when the structure is excited at the central peak antinode than at the nearby annular antinode. For the structure made by FIB, however, the CL intensity is similar when exciting at either antinode. This suggests that the constructive interference of SPP modes from different directions is improved in the EBL structures, leading to a stronger central peak.

Other features in the maps are also present off resonance. For instance, bright spots are present in the map of the FIB-milled structure [Figs. 7(d)-7(f)], which correspond to bright signals in the secondary electron image [Fig. 7(c)]. The groove edges are brighter in both structures regardless of wavelength. These correspond to topographic 
(a)

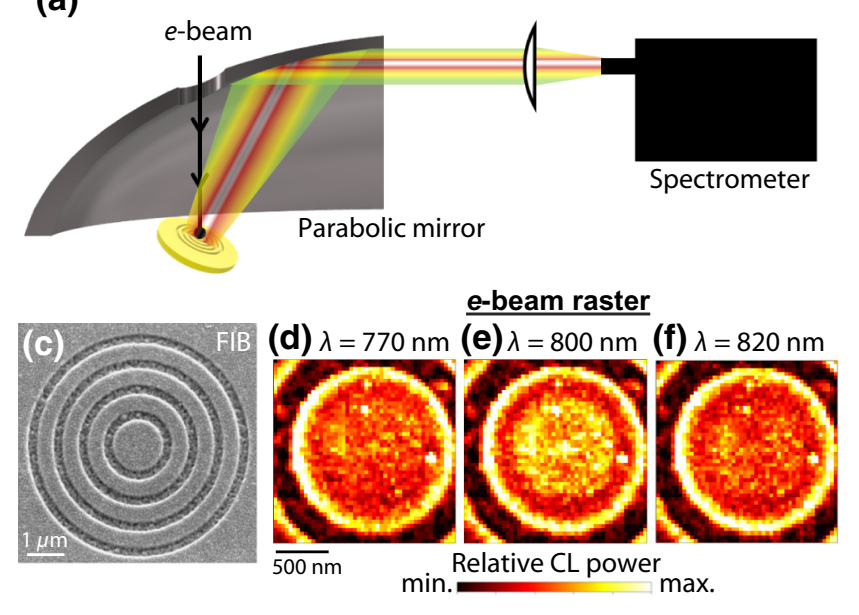

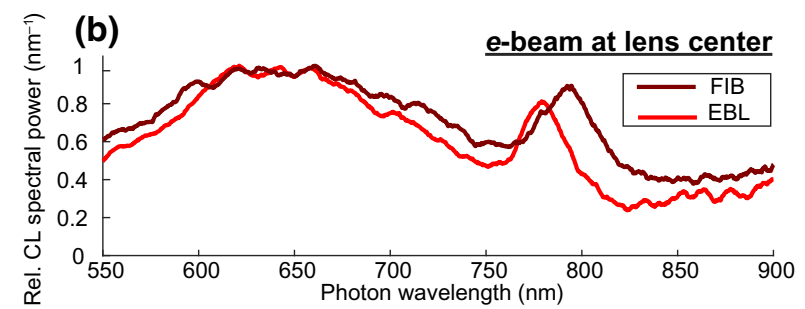

e-beam raster
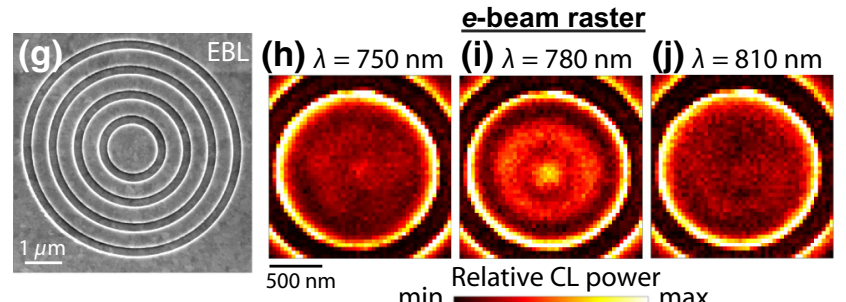

FIG. 7. Cathodoluminescence (CL) spectromicroscopy of the plasmonic resonance. (a) A schematic of the CL-spectromicroscopy technique. The electron-induced multicolor luminescence from a tilted lens is collected by a parabolic mirror and focused by a lens into a spectrometer. The electron beam is scanned and a full CL spectrum is collected at each beam position. (b) The average of the CL spectra collected within $75 \mathrm{~nm}$ of the structure center, shown for one lens made by FIB milling and one made EBL. (c) A SEM image and (d)-(f) CL spatial maps of the lens made by FIB. (g) A SEM image and (h)-(j) CL spatial maps of the lens made by EBL. The CL spatial maps are obtained from the spectromicroscopy data set by integrating over Gaussian wavelength bands with $2 \sigma=10 \mathrm{~nm}$. Each map is labeled with its corresponding center wavelength, $\lambda$.

features with locally high surface area and roughness, which can enhance the radiative LDOS by scattering more SPP modes out to light. The near-atomic smoothness of the central region in the EBL structures eliminates the scattering sites observed in the FIB structure. This will reduce damping of the resonance and thereby improve the field enhancement under laser illumination and consequently the amount of emitted electrons.

We note that there are key differences between CL mapping and the FDTD simulations used in Sec. II. The excitation mechanism is different: FDTD simulates a radially polarized laser excitation and calculates the field enhancement at all positions, whereas CL mapping rasters an e-beam and collects the integrated light output as a function of the e-beam position. This scanning local excitation provides a different spatial profile than expected for a global laser excitation based on FDTD. For instance, the CL emission is stronger when the electron beam is positioned at edges than at the structure center but this is not expected for the case of radially polarized illumination. Still, we can use CL mapping to visualize scattering sites and study the symmetry and smoothness of the plasmonicresonance mode, as discussed above. For more detailed discussion of the CL dependence on the electron-beam position, see the Appendix.

To quantify and compare the resonance characteristics of structures made by FIB and EBL, we fit the resonance peaks in the CL spectra collected using an ebeam at the lens center (where the plasmons generated match the circular symmetry of the structure) to determine the resonance wavelength and the FWHM. Average spectra from scan positions within $75 \mathrm{~nm}$ of the bullseye center are extracted from spectromicroscopy scans. The spectra are then rebinned into 2-nm wavelength bins and converted from the wavelength to the energy scale. The resonance peak is fitted using a Lorentzian plus a parabolic background. Four structures made by each fabrication method are measured and analyzed. The converted spectra and peak fitting are shown in Fig. 8. The average resonance wavelength is $798.4 \pm 8.8 \mathrm{~nm}$ standard deviation for the FIB-milled structures and $779.6 \pm 2.4 \mathrm{~nm}$ for the EBL structures. Both are near the 794-nm resonance wavelength predicted by FDTD for structures with the fabricated dimensions. The average measured frequencyto-FWHM ratio, or $Q$ factor, is $17.8 \pm 2.0$ for the FIBmilled structures and $23.9 \pm 1.9$ for the EBL structures. The more precise resonance wavelength and higher $Q$ factor (lower damping) of the EBL structures can be attributed to the reproducibility of the structure dimensions and their smoothness.

\section{Angle-resolved CL and photon-plasmon coupling}

We study the angle-resolved CL emission to infer the radial extent of effective plasmon propagation and grating coupling in the fabricated lenses. We both simulate and measure the CL far field for four- and 12-ring bullseye lenses. The far field is measured by positioning the electron beam at the structure center and imaging the parabolic mirror $[38,39]$. Because the emitting structure (several micrometers) is small compared to the distance from the mirror $(1 \mathrm{~mm})$, the position at which an emitted 

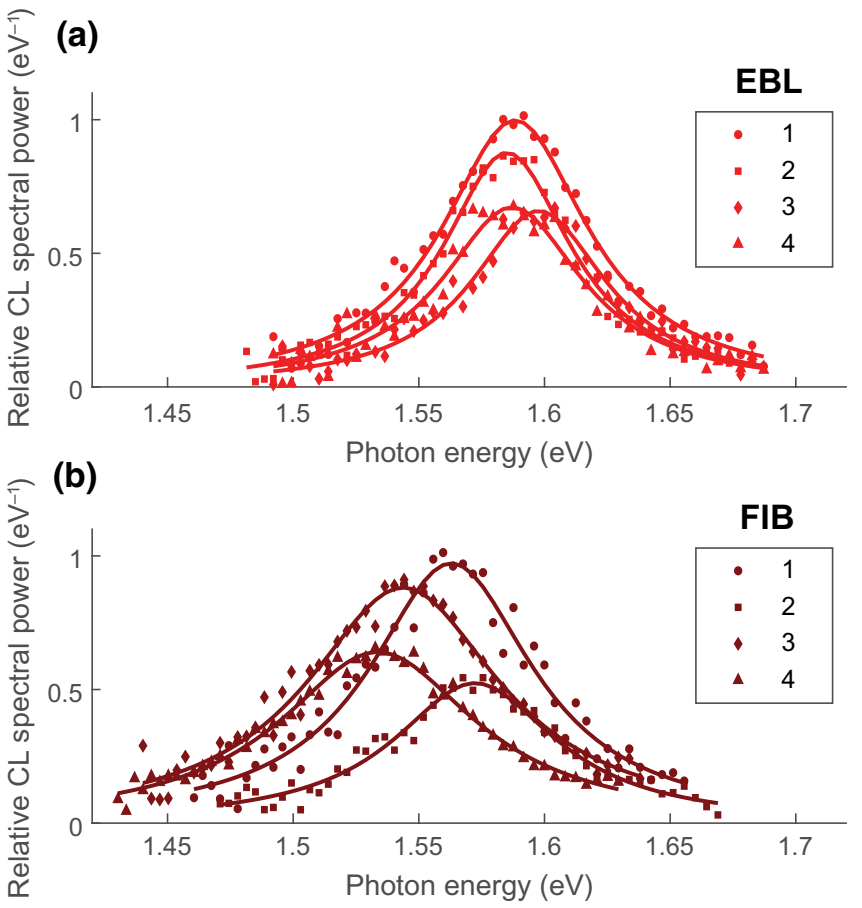

FIG. 8. A comparison of the plasmonic-resonance characteristics of bullseye lenses made by EBL and FIB milling using CL spectroscopy. Resonance peaks are shown for four lenses made using (a) EBL and (b) FIB. The spectra are fitted with a parabolic background plus a Lorentzian over a $0.2-\mathrm{eV}$ range centered at the peak. The points indicate data over the fitting window after the background is subtracted, while the lines indicate the Lorentzian peak-shape fit to the data.

photon reflects from the mirror is determined by the emission angle. The parabolic mirror brings the emission to infinity focus, which is filtered by a band-pass filter centered at $800 \mathrm{~nm}$ with $40-\mathrm{nm}$ bandwidth and then magnified and imaged onto a ThorLabs DCC3260M CMOS camera. For each measurement, 30 1-s exposures of the CCD are acquired and averaged. A background image with the beam blanked is acquired under the same conditions and is subtracted from the beam-on image. Then, each pixel in the image is mapped to an emission angle and the signal is normalized by the solid angle collected by that pixel to give an intensity map. The measured far field is then corrected for tilt and rotation of the sample relative to the mirror.

Using FDTD, the CL process can be numerically modeled [25]. An impinging 10-keV electron at the center of the bullseye is modeled using a series of dipoles normal to the surface. The dipoles are delayed in phase to create a propagating localized source of electric field. This generates a time- and $z$-dependent current density close to that of a moving electron [40]: $\vec{J}(t, z)=-e v \hat{z} \delta(z-v t) \delta(x-$ $\left.x_{0}\right) \delta\left(y-y_{0}\right)$. Here, $e$ is the fundamental charge, $v$ is the speed of the impinging electron, $\hat{z}$ is the unit vector in the $z$ direction, and $x_{0}$ and $y_{0}$ give the lateral position of the impinging electron. The resulting field decays laterally and vanishes within a few nanometers in the metal; therefore, no field can directly couple to the grooves and all observed emission is due to generation, propagation, and outcoupling of SPPs. To avoid the abrupt appearance and disappearance of the dipole field, which would create stray fields, a raised-cosine filter is used to gradually increase and decrease the amplitude oscillations of the starting and ending dipoles in time. The simulation box is gradually increased in order to collect larger angles and the mesh dimension is decreased to achieve convergence.

Simulated and measured far-field CL of four-ring lenses are shown in Figs. 9(a)-9(c). A donut beam is observed in all cases, supporting the proposition that the emission is radially polarized. Other work performing CL polarimetry on bullseyes has resolved the angle-dependent polarization
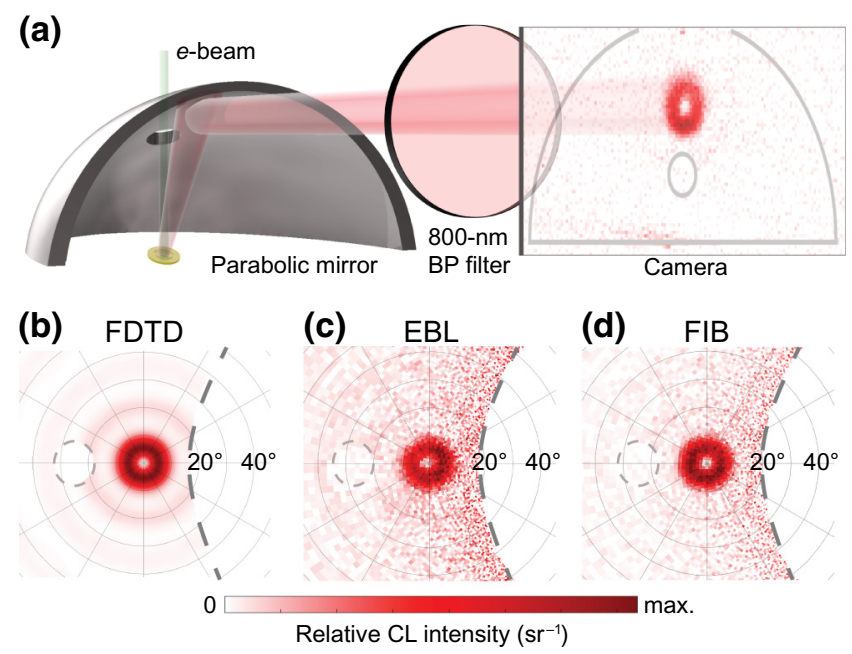

(e)

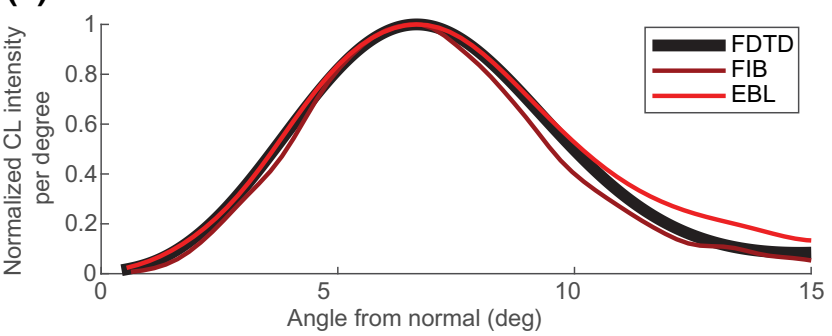

FIG. 9. Angle-resolved cathodoluminescence (CL) with e-beam at the structure center. (a) A schematic of the Fourier imaging technique. The parabolic mirror is imaged through a band-pass (BP) filter centered at 800-nm wavelength with 40-nm bandwidth. Far-field plots are then generated by transforming mirror coordinates to angular coordinates. The sample is tilted at $25^{\circ}$ so that emission is not lost through the entry hole for the electron beam. (b) The FDTD-simulated far field. CL far-field polar plots for lenses made using (c) EBL and (d) FIB. The gray dashed lines trace the electron-beam entry hole and the open face of the parabolic mirror. (e) CL distributions per degree from normal obtained by azimuthal integration of the far field. 
state and verified that the emission is radially polarized [41]. Both the angle of maximum emission and the overall angular distribution measured for lenses made by EBL and FIB closely match that predicted using FDTD [see Fig. 9(d)]. This indicates that the radial distribution of CL emission from plasmon-photon coupling is robust to fabrication imperfections, including the surface roughness of the FIBmilled structure. These measurements also show that this normally directed emission is captured effectively at $25^{\circ}$ sample tilt; this supports the validity of the spectra shown above and demonstrates the need to tilt the sample to study this emission.

We also fabricate and study a 12-ring bullseye lens using FIB as shown in Fig. 10(a). The simulated and measured far fields for this structure are shown in Figs. 10(b) and 10(c). Again, a donut beam is produced, as expected. The far field is more asymmetric than for the four-ring structures, which may be due to challenges in making the outer rings accurately concentric with the inner rings using FIB. Still, the angular breadth of emission is greatly reduced,

\section{(b)}
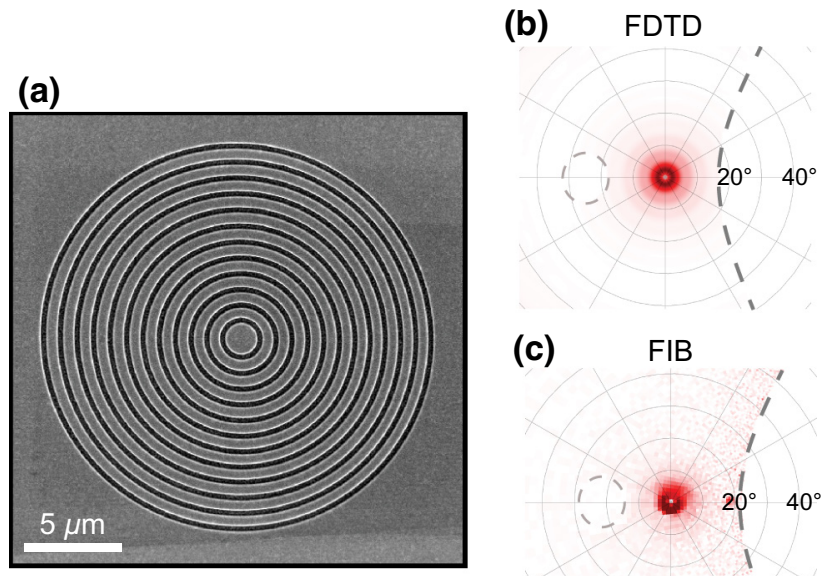

(c)
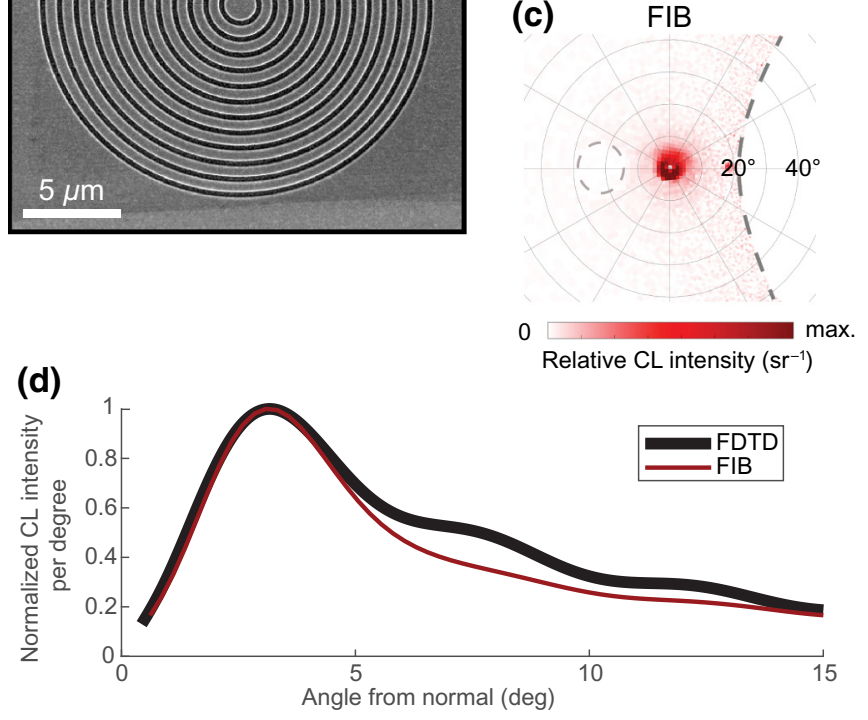

FIG. 10. Angle-resolved cathodoluminescence (CL) for a 12-ring lens milled using FIB. (a) A SEM image of the 12-ring lens. The FDTD-simulated (b) and experimentally measured (c) CL far-field polar maps for the electron beam positioned at the center. The gray dashed lines trace the electron-beam entry hole and the open face of the parabolic mirror. (d) The emission distribution along the angle from normal obtained by azimuthal integration of the far field. indicating that the plasmons can outcouple over a greater radial extent. As highlighted in Fig. 10(d), the peak emission angle is $3^{\circ}$, whereas in the four-ring lenses it is $7^{\circ}$. The distribution matches well between simulation and experiment overall, especially at less than $5^{\circ}$. This suggests that additional plasmon-propagation losses due to polycrystallinity are not a limiting factor even for this 12-ring structure.

\section{CONCLUSIONS}

We present a design for ultrafast nanoscale electron emitters, which has several advantages for the next generation of electron-based instrumentation. First, the design is compatible with high accelerating fields, while decreasing the source size by 2 orders of magnitude as compared to an unpatterned flat cathode. Electromagnetic simulations and cathodoluminescence mapping support the notion that plasmonic resonance leads to a single dominant central peak in optical intensity, which can be used to generate electron pulses with $140-\mathrm{nm}$ FWHM (60-nm rms) lateral size. Second, the emission surface can be made nearly atomically flat, suppressing emittance increase from surface roughness and from aberrations due to field curvature, differently from what happens with tips. Third, the geometric parameters can be tuned to optimize the photocurrent and temporal response for application requirements without changing the emission spot size. The computations and experiments shown here support the proposition that the plasmonic properties and the spectral bandwidth of such structures are compatible with the emission of sub-10-fs pulses.

These plasmonic lenses could also facilitate emerging high-intensity modes of operation. For instance, they reduce the laser power required to access the opticalfield emission regime, in which the fields are strong enough to modulate the work function at the optical frequency. This operating regime is of great interest because it allows control of the photocurrent density at attosecond time scales, providing the potential to generate attosecond electron-pulse trains [42]. In addition, the compatibility of these lenses with few-cycle pulses could allow control of the photocurrent intensity by tuning the carrier envelope phase, as has been demonstrated for tip emitters [43].

In terms of applications, the transverse brightness of such nanocathodes is expected to be more than one order of magnitude better than present state-of-the-art flat cathodes. Their use in rf environments would enable production of relativistically accelerated electrons with picometer emittance, which could be focused down to nanometer sizes and efficiently injected into advanced acceleration devices [44]. In ultrafast electron imaging, such sources promise to bridge the gap in spatial resolution between static and ultrafast relativistic sources, applying electron-based characterization to nanoscale dynamics. 


\section{ACKNOWLEDGMENTS}

D.F. and the work at the Molecular Foundry were supported by the Office of Science, Office of Basic Energy Sciences, of the U.S. Department of Energy under Contract No. DE-AC02-05CH11231. Funding for D.B.D. was provided by STROBE: A National Science Foundation Science and Technology Center under Grant No. DMR 1548924. We thank F. Ogletree, S. Aloni, and E. S. Barnard at the Molecular Foundry for their advice and assistance with the CL setup. We thank S. Dhuey at the Molecular Foundry for assistance with the e-beam lithography.

D.B.D. and F.R. contributed equally to this work.

\section{APPENDIX: CL DEPENDENCE ON ELECTRON-BEAM POSITION}

As discussed in Sec. IV, scanning CL can be used to obtain qualitative maps of the plasmonic resonance and, more generally, the radiative LDOS. These are fundamentally different from the field-enhancement profile expected upon excitation with a radially polarized beam. The main difference is that the electron beam only excites purely radial plasmon modes in the structure when the beam is positioned at the center. Elsewhere, the beam generates SPPs traveling outward from its current position rather than the lens center, so the modes excited do not match the circular symmetry of the lens. This plasmonic configuration would not be excited by a radially polarized laser.

Using angle-resolved CL, we can observe this symmetry mismatch between plasmons and the lens for off-center electron-beam positions, shown in Fig. 11 for a fourring lens. Instead of a circularly symmetric donut-shaped emission, complex interference patterns are generated in the far field, the shape of which depends on the beam position [41].

For this reason, it is hard to quantitatively compare spatial profiles from CL spectromicroscopy and simulated field enhancement under laser illumination. One example of this is the observation that the CL emission is brighter when the beam is at the groove edge than at the center of the structure (see Fig. 7). At the edge, the plasmon modes excited and how they couple to the structure are very different than when the beam is at the center, as evidenced by the far field shown in Figs. 11(d) and 11(f). Also, the edge is a strong scattering site, allowing many SPP modes generated nearby to couple to light, giving an enhanced radiative LDOS. Therefore, we still expect that the field enhancement and consequent photoemission under laser illumination will be stronger at the center than the edges.

Quantitative analysis of the spatial map is further complicated by the nature of the CL process. The total measured intensity of this emission depends on how the emission interferes in the far field and what parts of the emission are collected by the mirror. At both the node and

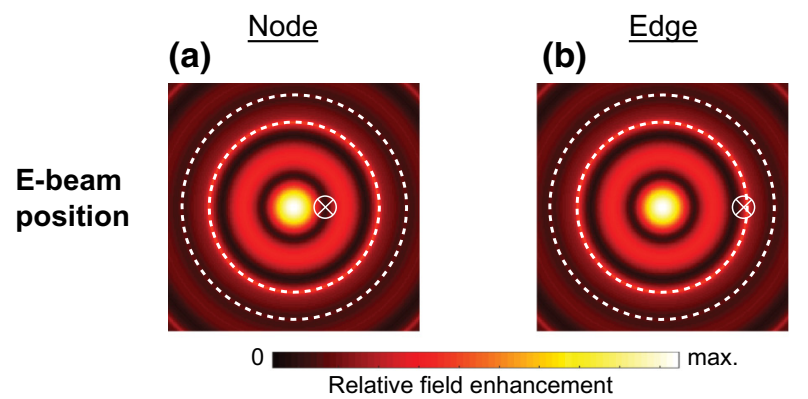

(c)

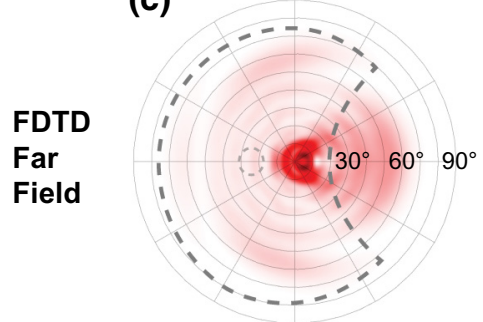

(e)

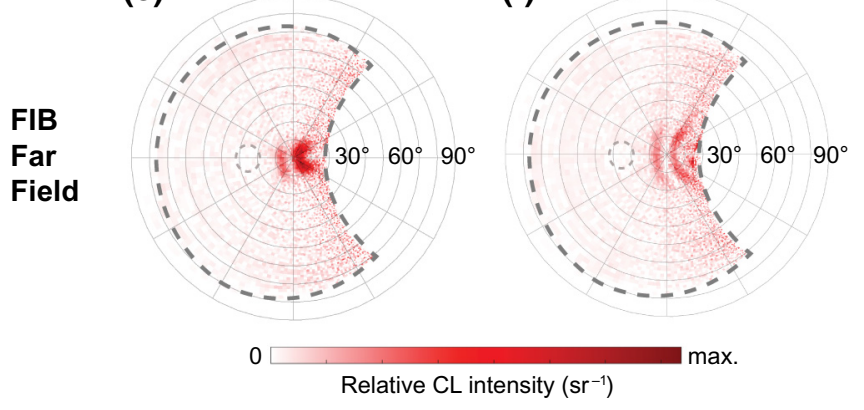

FIG. 11. Angle-resolved cathodoluminescence (CL) for a four-ring lens when the electron beam is positioned off center. (a),(b) The electron-beam position (cross symbol) superimposed on the FDTD-simulated field-enhancement profile for a radially polarized beam with $800-\mathrm{nm}$ wavelength. The dashed circles indicate the edges of the first groove. (c),(d) FDTD-simulated far-field polar maps for the electron beam at the node and edge positions. The gray dashed lines trace the electron-beam entry hole and the open face of the parabolic mirror. (e),(f) Measured $\mathrm{CL}$ far-field polar maps at these positions for a lens fabricated using FIB.

edge positions, some of the emitted light is not collected by the mirror [see Figs. 11(c)-11(f)]. Still, a valuable qualitative insight can be obtained from scanning $\mathrm{CL}$ as discussed in Sec. IV B, such as visualizing the plasmonic-resonance mode and nanoscale heterogeneities.

[1] Bradley J. Siwick, Jason R. Dwyer, Robert E. Jordan, and R. J. Dwayne Miller, An atomic-level view of melting using femtosecond electron diffraction, Science 302, 1382 (2003).

[2] Vance R. Morrison, Robert P. Chatelain, Kunal L. Tiwari, Ali Hendaoui, Andrew Bruhács, Mohamed Chaker, and Bradley J. Siwick, A photoinduced metal-like phase of 
monoclinic $\mathrm{VO}_{2}$ revealed by ultrafast electron diffraction, Science 346, 445 (2014).

[3] Maximilian Eichberger, Hanjo Schäfer, Marina Krumova, Markus Beyer, Jure Demsar, Helmuth Berger, Gustavo Moriena, Germán Sciaini, and R. J. Dwayne Miller, Snapshots of cooperative atomic motions in the optical suppression of charge density waves, Nature 468, 799 (2010).

[4] Ahmed H. Zewail, 4D ultrafast electron diffraction, crystallography, and microscopy, Annu. Rev. Phys. Chem. 57, 65 (2006).

[5] Meng Gao, Cheng Lu, Hubert Jean-Ruel, Lai Chung Liu, Alexander Marx, Ken Onda, Shin-ya Koshihara, Yoshiaki Nakano, Xiangfeng Shao, Takaaki Hiramatsu, et al., Mapping molecular motions leading to charge delocalization with ultrabright electrons, Nature 496, 343 (2013).

[6] M. J. Rhee, Refined definition of the beam brightness, Phys. Fluids B: Plasma Phys. 4, 1674 (1992).

[7] F. Carbone, P. Musumeci, O. J. Luiten, and C. Hebert, A perspective on novel sources of ultrashort electron and X-ray pulses, Chem. Phys. 392, 1 (2012).

[8] Armin Feist, Nara Rubiano da Silva, Wenxi Liang, Claus Ropers, and Sascha Schäfer, Nanoscale diffractive probing of strain dynamics in ultrafast transmission electron microscopy, Struct. Dyn. 5, 014302 (2018).

[9] Nara Rubiano da Silva, Marcel Möller, Armin Feist, Henning Ulrichs, Claus Ropers, and Sascha Schäfer, Nanoscale Mapping of Ultrafast Magnetization Dynamics with Femtosecond Lorentz Microscopy, Phys. Rev. X 8, 031052 (2018).

[10] Aycan Yurtsever, Renske M. van der Veen, and Ahmed H. Zewail, Subparticle ultrafast spectrum imaging in 4D electron microscopy, Science 335, 59 (2012).

[11] Jared Maxson, David Cesar, Giacomo Calmasini, Alexander Ody, Pietro Musumeci, and David Alesini, Direct Measurement of Sub-10 fs Relativistic Electron Beams with Ultralow Emittance, Phys. Rev. Lett. 118, 154802 (2017).

[12] Lingrong Zhao et al., Terahertz Streaking of FewFemtosecond Relativistic Electron Beams, Phys. Rev. X 8, 021061 (2018).

[13] R. Huang, D. Filippetto, C. F. Papadopoulos, H. Qian, F. Sannibale, and M. Zolotorev, Dark current studies on a normal-conducting high-brightness very-high-frequency electron gun operating in continuous wave mode, Phys. Rev. Spec. Top. Accel. Beams 18, 013401 (2015).

[14] Aleksandr Polyakov, Christoph Senft, K. F. Thompson, J. Feng, S. Cabrini, P. J. Schuck, H. A. Padmore, Samuel J. Peppernick, and Wayne P. Hess, Plasmon-Enhanced Photocathode for High Brightness and High Repetition Rate X-Ray Sources, Phys. Rev. Lett. 110, 076802 (2013).

[15] R. K. Li, H. To, G. Andonian, J. Feng, A. Polyakov, C. M. Scoby, K. Thompson, W. Wan, H. A. Padmore, and P. Musumeci, Surface-Plasmon Resonance-Enhanced Multiphoton Emission of High-Brightness Electron Beams from a Nanostructured Copper Cathode, Phys. Rev. Lett. 110, 074801 (2013).

[16] Y. Y. Lau, Effects of cathode surface roughness on the quality of electron beams, J. Appl. Phys. 61, 36 (1987).

[17] Jennifer M. Steele, Zhaowei Liu, Yuan Wang, and Xiang Zhang, Resonant and non-resonant generation and focusing of surface plasmons with circular gratings, Opt. Express 14, 5664 (2006).
[18] Weibin Chen, Don C. Abeysinghe, Robert L. Nelson, and Qiwen Zhan, Plasmonic lens made of multiple concentric metallic rings under radially polarized illumination, Nano Lett. 9, 4320 (2009).

[19] J. H. Bechtel, W. Lee Smith, and N. Bloembergen, Twophoton photoemission from metals induced by picosecond laser pulses, Phys. Rev. B 15, 4557 (1977).

[20] Andrei Kolomenski, Alexandre Kolomenskii, John Noel, Siying Peng, and Hans Schuessler, Propagation length of surface plasmons in a metal film with roughness, Appl. Opt. 48, 5683 (2009).

[21] M. Kuttge, E. J. R. Vesseur, J. Verhoeven, H. J. Lezec, H. A. Atwater, and A. Polman, Loss mechanisms of surface plasmon polaritons on gold probed by cathodoluminescence imaging spectroscopy, Appl. Phys. Lett. 93, 113110 (2008).

[22] Kevin M. McPeak, Sriharsha V. Jayanti, Stephan J. P. Kress, Stefan Meyer, Stelio Iotti, Aurelio Rossinelli, and David J. Norris, Plasmonic films can easily be better: Rules and recipes, ACS Photonics 2, 326 (2015).

[23] Robert L. Olmon, Brian Slovick, Timothy W. Johnson, David Shelton, Sang-Hyun Oh, Glenn D. Boreman, and Markus B. Raschke, Optical dielectric function of gold, Phys. Rev. B 86, 235147 (2012).

[24] Heinz Raether, in Surface Plasmons on Smooth and Rough Surfaces and on Gratings (Springer, 1988), p. 91.

[25] LUMERICAL, FDTD solutions, http://www.lumerical.com.

[26] M. A. Laughton and D. F. Warne, Electrical Engineer's Reference Book (Elsevier, 2003), 16th ed., p. 384.

[27] Tineke Thio, K. M. Pellerin, R. A. Linke, H. J. Lezec, and T. W. Ebbesen, Enhanced light transmission through a single subwavelength aperture, Opt. Lett. 26, 1972 (2001).

[28] Ian R. Hooper and J. Roy Sambles, Dispersion of surface plasmon polaritons on short-pitch metal gratings, Phys. Rev. B 65, 165432 (2002).

[29] Nicolas Vogel, Julius Zieleniecki, and Ingo Köper, As flat as it gets: Ultrasmooth surfaces from template-stripping procedures, Nanoscale 4, 3820 (2012).

[30] F. J. García De Abajo, Optical excitations in electron microscopy, Rev. Mod. Phys. 82, 209 (2010).

[31] Martin Kuttge, Ernst Jan R. Vesseur, A. F. Koenderink, H. J. Lezec, H. A. Atwater, F. J. García de Abajo, and Albert Polman, Local density of states, spectrum, and farfield interference of surface plasmon polaritons probed by cathodoluminescence, Phys. Rev. B 79, 113405 (2009).

[32] Carrie E. Hofmann, Ernst Jan R. Vesseur, Luke A. Sweatlock, Henri J. Lezec, F. Javier García de Abajo, Albert Polman, and Harry A Atwater, Plasmonic modes of annular nanoresonators imaged by spectrally resolved cathodoluminescence, Nano Lett. 7, 3612 (2007).

[33] Arthur Losquin and Mathieu Kociak, Link between cathodoluminescence and electron energy loss spectroscopy and the radiative and full electromagnetic local density of states, ACS Photonics 2, 1619 (2015).

[34] Toon Coenen, Benjamin J. M. Brenny, Ernst Jan Vesseur, and Albert Polman, Cathodoluminescence microscopy: Optical imaging and spectroscopy with deep-subwavelength resolution, MRS Bull. 40, 359 (2015).

[35] Mathieu Kociak, Odile Stéphan, Alexandre Gloter, Luiz F. Zagonel, Luiz H. G. Tizei, Marcel Tencé, Katia March, Jean Denis Blazit, Zackaria Mahfoud, Arthur Losquin, et al., Seeing and measuring in colours: Electron microscopy and 
spectroscopies applied to nano-optics, C.R. Phys. 15, 158 (2014).

[36] Daniel B. Durham, D. Frank Ogletree, and Edward S. Barnard, Scanning Auger spectromicroscopy using the SCOPEFOUNDRY software platform, Surf. Interface Anal. 50, 1174 (2018).

[37] Edward S. Barnard, SCOPEFOUNDRY: A PYTHON platform for controlling custom laboratory experiments and visualizing scientific data, http://www.scopefoundry.org/.

[38] Toon Coenen, Ernst Jan R. Vesseur, and Albert Polman, Angle-resolved cathodoluminescence spectroscopy, Appl. Phys. Lett. 99, 143103 (2011).

[39] Toon Coenen et al., Angle-Resolved Cathodoluminescence Nanoscopy (Universiteit van Amsterdam [Host], 2014).

[40] Pratik Chaturvedi, Keng H. Hsu, Anil Kumar, Kin Hung Fung, James C. Mabon, and Nicholas X. Fang, Imaging of plasmonic modes of silver nanoparticles using high-resolution cathodoluminescence spectroscopy, ACS Nano 3, 2965 (2009).

[41] Clara I. Osorio, Toon Coenen, Benjamin J. M. Brenny, Albert Polman, and A. Femius Koenderink, Angle-resolved cathodoluminescence imaging polarimetry, ACS Photonics 3, 147 (2015).

[42] Michael Krüger, Markus Schenk, Michael Förster, and Peter Hommelhoff, Attosecond physics in photoemission from a metal nanotip, J. Phys. B: At. Mol. Opt. Phys. 45, 074006 (2012).

[43] Björn Piglosiewicz, Slawa Schmidt, Doo Jae Park, Jan Vogelsang, Petra Groß, Cristian Manzoni, Paolo Farinello, Giulio Cerullo, and Christoph Lienau, Carrier-envelope phase effects on the strong-field photoemission of electrons from metallic nanostructures, Nat. Photonics 8, 37 (2014).

[44] R. Joel England et al., Dielectric laser accelerators, Rev. Mod. Phys. 86, 1337 (2014). 